\title{
A LOWER BOUND FOR THE DIAMETER OF SOLUTIONS TO THE RICCI FLOW WITH NONZERO $H^{1}\left(M^{n} ; \mathbb{R}\right)$
}

\author{
Tom Ilmanen And Dan Knopf
}

\section{Introduction}

Consider the Ricci flow

$$
\frac{\partial}{\partial t} g=-2 \operatorname{Rc}(g), \quad 0 \leq t<T,
$$

of a metric $g$ on $S^{1} \times S^{2}$. Our intuition suggest that no matter how wild the metric is, the Ricci curvature in the $S^{1}$ direction should more or less average out to zero, so that the distance around the $S^{1}$ should tend not to decrease.

In this paper, we substantiate this idea by proving the following more general theorem. Given a Riemannian manifold $\left(M^{n}, g\right)$ and a homology element $\alpha \in$ $H_{1}\left(M^{n} ; \mathbb{Z}\right)$, let $L_{\alpha}(g)$ denote the infimum of the lengths measured with respect to $g$ of all curves representing $\alpha$.

Theorem 1. If $\left(M^{n}, g(t): 0 \leq t<T\right)$ is a compact solution of the Ricci flow and $\alpha \in H_{1}\left(M^{n} ; \mathbb{Z}\right)$ is an element of infinite order, there exists $c=$ $c(\alpha, g(0))$ such that

$$
L_{\alpha}(g(t)) \geq c>0
$$

for all $t \in[0, T)$.

A particular consequence is that the diameter of $\left(M^{n}, g(t)\right)$ is bounded from below independently of $t$. As a result, we can resolve a conjecture made by Hamilton in $\S 26$ of [4]. Suppose that $\left(M^{n}, g(t): 0 \leq t<T\right)$ is a solution of the Ricci flow on a maximal time interval. For $x_{j} \in M^{n}, t_{j} \in[0, T)$, and $\lambda_{j}>0$, define the dilations

$$
g_{j}(t):=\lambda_{j} g\left(t_{j}+\frac{t}{\lambda_{j}}\right), \quad-\lambda_{j} t_{j} \leq t<\lambda_{j}\left(T-t_{j}\right) .
$$

If $t_{j} \nearrow T$ as $j \rightarrow \infty$ and $\left(M^{n}, g_{j}(t), x_{j}\right)$ converges locally smoothly to a limit $\left(M_{\infty}^{n}, g_{\infty}(t), x_{\infty}\right)$, we call the latter a final time limit flow of $\left(M^{n}, g(t): 0 \leq t<\right.$ $T)$. The following result answers Hamilton's conjecture affirmatively.

Corollary 1. $\left(S^{1} \times S^{n-1}, \bar{g}(t)\right)$ cannot arise as a final time limit flow.

Received October 3, 2002.

Second author partially supported by NSF grant DMS - 0202796. 
Here $\bar{g}(t)$ is the Ricci soliton

$$
\bar{g}(t):=d s \otimes d s+2(n-1)(\bar{T}-t) g_{\mathrm{can}},
$$

where $g_{\text {can }}$ is a round metric on $S^{n-1}$. Note that the only possible final time limit flow of $\left(S^{1} \times S^{n-1}, \bar{g}(t)\right)$ is $\left(\mathbb{R} \times S^{n-1}, \bar{g}(t)\right)$. The main content of the corollary concerns the case that only a subsequence is known to converge.

In order to put our results into context, recall that we should not be surprised if a solution $\left(M^{n}, g(t)\right)$ of the Ricci flow starting from an arbitrary Riemannian manifold encounters a finite time singularity. Indeed, this must be the case if the scalar curvature ever becomes everywhere positive. (See the proof of Corollary 1, below.) To study a finite time singularity, it is often useful to construct a sequence of dilations (1.2), sometimes called a blowup sequence. In certain cases (namely if the $\lambda_{j}$ are comparable to the suprema of the curvatures and if an injectivity radius estimate is available for the sequence $g_{j}$ ) one can apply Gromov-type compactness arguments such as those in [5] in order to show $C^{\infty}$ convergence to a final time limit flow. (Such flows are also called singularity models in the literature.) Final time limit flows have special properties which aid analysis of the original solution $\left(M^{n}, g(t)\right)$. With sufficient knowledge of the limit, one can draw useful conclusions about the analytic, geometric, and topological character of a singular solution just prior to the formation of the singularity. The analysis of singularities via the formation of final time limit flows is an integral part of Hamilton's well-developed program to resolve Thurston's Geometrization Conjecture for closed 3-manifolds [7] by means of the Ricci flow. (See for example [4] and the survey [1].)

Theorem 1 is essentially a monotonicity result. We shall offer two proofs which are dual to one another. The first proof (Section 2) uses cohomology, is simpler, and is more direct. The second proof (Section 3) uses homology. Its value lies in better revealing the geometry; in particular; we hope that it is instructive in showing how the ideas introduced here might be generalized. We briefly discuss such potential applications in Section 4 .

\section{The cohomology proof}

Our starting point is the following observation, which was pointed out to the first author by Sun-Chin (Michael) Chu [2]. Let $\left(M^{n}, g(t)\right)$ be a solution of the Ricci flow, and let $\phi(t)$ be a 1-parameter family of 1-forms evolving by

$$
\frac{\partial}{\partial t} \phi=\Delta_{d} \phi
$$

where $-\Delta_{d}:=d \delta+\delta d$ is the Hodge-de Rham Laplacian. Recalling that

$$
\Delta_{d} \phi_{i}=\Delta \phi_{i}-R_{i}^{j} \phi_{j}
$$


where $\Delta$ is the rough Laplacian, one computes that

$$
\begin{aligned}
\frac{\partial}{\partial t}|\phi|^{2} & =\frac{\partial}{\partial t}\left(g^{i j} \phi_{i} \phi_{j}\right) \\
& =2 R^{i j} \phi_{i} \phi_{j}+2 \phi^{i} \frac{\partial}{\partial t} \phi_{i} \\
& =2 \phi^{i} \Delta \phi_{i} \\
& =\Delta|\phi|^{2}-2|\nabla \phi|^{2} .
\end{aligned}
$$

Applying the parabolic maximum principle, one concludes that

$$
\|\phi(t)\|_{g(t)} \leq\|\phi(0)\|_{g(0)}
$$

for as long as the solution $g(t)$ exists, where $\|\phi\|_{g}$ denotes the supremum norm $\|\phi\|_{g}:=\sup _{x \in M}|\phi(x)|_{g(x)}$.

We use this observation to establish a key monotonicity property. Given a Riemannian manifold $\left(M^{n}, g\right)$ and an element $\Phi$ of the first de Rham cohomology group $H_{d R}^{1}\left(M^{n} ; \mathbb{R}\right)$, define

$$
N_{g}(\Phi):=\inf _{\phi \in \Phi}\|\phi\|_{g} .
$$

Lemma 1. If $\left(M^{n}, g(t): 0 \leq t<T\right)$ is a solution of the Ricci flow, $N_{g(t)}(\Phi)$ is a non-increasing function of time.

Proof. For any $\varepsilon>0$, there is a smooth representative $\phi_{0} \in \Phi$ such that

$$
\left\|\phi_{0}\right\| \leq N_{g(0)}(\Phi)+\varepsilon \text {. }
$$

Define $\phi(t)$ by

$$
\begin{aligned}
\frac{\partial}{\partial t} \phi & =\Delta_{d} \phi \\
\phi(0) & =\phi_{0},
\end{aligned}
$$

noting that a solution $\phi(t)$ exists for as long as $g(t)$ exists. Note too that $\phi(t) \in \Phi$. Indeed, if we define a smooth function $F(t)$ by

$$
\begin{aligned}
\frac{\partial F}{\partial t} & =\Delta F-\delta \phi_{0} \\
F(0) & =0,
\end{aligned}
$$

we have

$$
\phi=\phi_{0}+d F
$$

for all $t \in[0, T)$, because

$$
\frac{\partial}{\partial t}\left(\phi_{0}+d F\right)=\Delta_{d}\left(\phi_{0}+d F\right)
$$

Hence by $(2.1)$, we obtain

$$
N_{g(t)}(\Phi) \leq\|\phi\|_{g(t)} \leq\|\phi\|_{g(0)} \leq N_{g(0)}(\Phi)+\varepsilon .
$$


The following observation is of independent interest.

Lemma 2. If $\left(M^{n}, g\right)$ is a compact Riemannian manifold, then $N_{g}$ is a norm on $H_{d R}^{1}\left(M^{n} ; \mathbb{R}\right)$.

Proof. Homogeneity and the triangle inequality are readily verified. To show positivity, suppose that $N_{g}(\Phi)=0$. Then there is a sequence $\left\{\phi_{j}: j \in \mathbb{N}\right\} \subset \Phi$ of smooth 1-forms such that $\left\|\phi_{j}\right\|_{g} \rightarrow 0$. Fix any $\phi \in \Phi$. We may write

$$
\phi-\phi_{j}=d F_{j},
$$

where each $F_{j}$ is smooth. Since $M^{n}$ is compact, $\sup _{j \in \mathbb{N}}\left\|d F_{j}\right\|<\infty$. So after adding a locally constant function to $F_{j}$, we may by Arzela-Ascoli select a subsequence $F_{j_{k}}$ that converges uniformly to a Lipschitz function $F$. Then

$$
\text { ess sup }|\phi-d F|_{g} \leq \limsup _{k \rightarrow \infty}\left\|\phi-d F_{j_{k}}\right\|_{g}=\limsup _{k \rightarrow \infty}\left\|\phi_{j_{k}}\right\|_{g}=0 .
$$

So $d F=\phi$ almost everywhere, which implies in particular that $F$ is smooth. Hence $\Phi=0$.

We can now obtain a lower bound for the diameter of a solution of the Ricci flow on a compact manifold $M^{n}$ with $H^{1}\left(M^{n} ; \mathbb{R}\right) \neq\{0\}$.

First proof of Theorem 1. Let $\left(M^{n}, g(t): 0 \leq t<T\right)$ be a solution of the Ricci flow. Consider the natural map $\rho: H_{1}\left(M^{n} ; \mathbb{Z}\right) \rightarrow H_{1}\left(M^{n} ; \mathbb{R}\right)$ and note that $\beta \in H_{1}\left(M^{n} ; \mathbb{Z}\right)$ is a torsion element if and only if $\rho(\beta)=0$, hence if and only if $\langle\Psi, \beta\rangle=0$ for all $\Psi \in H^{1}\left(M^{n} ; \mathbb{R}\right)$. So if $\alpha \in H_{1}\left(M^{n} ; \mathbb{Z}\right)$ is an element of infinite order, then there exists $\Phi \in H^{1}\left(M^{n} ; \mathbb{R}\right)$ such that $\langle\Phi, \alpha\rangle>0$.

Fix any $t \in[0, T)$, and let $a$ be any curve representing $\alpha$. Then for all $\phi \in \Phi$, we have

$$
0<\langle\Phi, \alpha\rangle=\int_{a} \phi \leq\|\phi\|_{g(t)} \cdot \operatorname{length}_{g(t)}(a) .
$$

Taking the infimum over $\phi \in \Phi$, we get

$$
\langle\Phi, \alpha\rangle \leq N_{g(t)}(\Phi) \cdot \operatorname{length}_{g(t)}(a) \leq N_{g(0)}(\Phi) \cdot \operatorname{length}_{g(t)}(a)
$$

by Lemma 1. Taking the infimum over all $a \in \alpha$, we obtain

$$
L_{\alpha}(g(t)) \geq \frac{\langle\Phi, \alpha\rangle}{N_{g(0)}(\Phi)}>0 .
$$

Proof of Corollary 1. Let $\left(M^{n}, g(t): 0 \leq t<T \leq \infty\right)$ be a solution of the Ricci flow on a maximal time interval, and let $g_{j}(t)=\lambda_{j} g\left(t_{j}+t / \lambda_{j}\right)$ be a sequence of dilations such that

$$
\left(M^{n}, g_{j}(t)\right) \rightarrow\left(S^{1} \times S^{n-1}, \bar{g}(t)\right),
$$

where $\bar{g}(t)$ is defined by (1.3). Then there exists $j_{0}$ such that $g\left(t_{j_{0}}\right)$ has positive scalar curvature $R>0$. Because

$$
\frac{\partial}{\partial t} R=\Delta R+2|\mathrm{Rc}|^{2} \geq \Delta R+\frac{2}{n} R^{2},
$$


the maximum principle implies that the solution must fail to exist at a finite time $T<\infty$. By Theorem 8.1 of [4], a finite time singularity implies that

$$
\limsup _{t \nearrow T}\left(\sup _{x \in M}|\operatorname{Rm}(x, t)|\right)=\infty .
$$

Then because there is $C=C(n)$ such that

$$
\frac{\partial}{\partial t}|\mathrm{Rm}|^{2} \leq \Delta|\mathrm{Rm}|^{2}+C|\mathrm{Rm}|^{3},
$$

the maximum principle further implies a lower bound for the curvature blowup rate,

$$
\sup _{x \in M}|\operatorname{Rm}(x, t)| \geq \frac{2 / C}{T-t} .
$$

But then smooth convergence (2.5) is possible only if

$$
\lim _{j \rightarrow \infty} \lambda_{j}=\infty \text {. }
$$

On the other hand, since $S^{1} \times S^{n-1}$ is compact, (2.5) also implies that

$$
H_{1}\left(M^{n} ; \mathbb{Z}\right) \cong H_{1}\left(S^{1} \times S^{n-1} ; \mathbb{Z}\right) \cong \mathbb{Z} .
$$

Let $\alpha$ generate $H_{1}\left(M^{n} ; \mathbb{Z}\right)$. By Theorem 1 , we have

$$
L_{\alpha}(g(t)) \geq c>0 \text {. }
$$

Hence

$$
L_{\alpha}\left(g_{j}(0)\right) \geq \sqrt{\lambda_{j}} c \rightarrow \infty
$$

as $j \rightarrow \infty$. This contradicts (2.5) and establishes Corollary 1.

\section{The homology proof}

We now seek a monotone quantity dual to the metric norms $N_{g(t)}$ defined above on $H_{d R}^{1}$. Let $\left(M^{n}, g\right)$ be a Riemannian manifold. For each free homotopy class $\Gamma \in \operatorname{Free}\left(M^{n}\right)$, define

$$
\begin{aligned}
\ell_{g}(\Gamma) & :=\inf _{\gamma \in \Gamma} \operatorname{length}_{g}(\gamma), \\
m_{g}(\Gamma) & :=\liminf _{k \rightarrow \infty} \frac{\ell_{g}(k \Gamma)}{k},
\end{aligned}
$$

where $k \Gamma$ denotes the $k$-fold cover of $\Gamma$.

We first obtain a lower bound on the decay of $\ell_{g(t)}(\Gamma)$ during the Ricci flow.

Lemma 3. Let $\left(M^{n}, g(t): 0 \leq t<T\right)$ be a solution of the Ricci flow and $\Gamma \in \operatorname{Free}\left(M^{n}\right)$ a free homotopy class. Then there exists $C>0$ depending only on $n$ such that

$$
\left(\ell_{g(t)}(\Gamma)\right)^{2} \geq\left(\ell_{g(0)}(\Gamma)\right)^{2}-C t
$$

for all $t \in[0, T)$. 
Proof. We may assume $\Gamma$ is nontrivial. Fix $t \in[0, T)$. There is a nontrivial smooth closed geodesic $\gamma \in \Gamma$ such that

$$
\text { length }_{g(t)}(\gamma)=\ell_{g(t)}(\Gamma)>0 \text {. }
$$

Let $V$ denote the unit tangent vector field along $\gamma$. Stability implies that

$$
\int_{\gamma}\left(\left|\nabla_{V} X\right|^{2}-\langle R(V, X) X, V\rangle\right) d s \geq 0
$$

for any smooth vector field $X$ along $\gamma$. Because of holonomy, there may not exist a parallel orthonormal frame along $\gamma$; but we can choose an orthonormal frame $\left(e_{1}, \ldots, e_{n}\right)$ along $\gamma$ such that $e_{n}=V$ and

$$
\left|\nabla_{V} e_{i}\right| \leq \frac{C_{n}}{\operatorname{length}_{g(t)}\left(\gamma_{t}\right)}=\frac{C_{n}}{\ell_{g(t)}(\Gamma)}
$$

for $1 \leq i \leq n-1$, where $C_{n}>0$ depends only on $n$. Taking $X=e_{i}$ in (3.1) and summing over $i=1, \ldots, n-1$ yields

$$
0 \leq(n-1)\left(\frac{C_{n}}{\operatorname{length}_{g(t)}(\gamma)}\right)^{2} \cdot \operatorname{length}_{g(t)}(\gamma)-\int_{\gamma} \operatorname{Rc}(V, V) d s
$$

Thus

$$
\left.\frac{d}{d s}\left(\operatorname{length}_{g(s)}(\gamma)\right)\right|_{s=t}=-\int_{\gamma} \operatorname{Rc}(V, V) d s \geq-\frac{(n-1) C_{n}^{2}}{\operatorname{length}_{g(t)}(\gamma)} .
$$

Now define

$$
\begin{aligned}
f & : \Gamma \times[0, T) \rightarrow \mathbb{R}, \\
f(\beta, t) & :=\operatorname{length}_{g(t)}(\beta) .
\end{aligned}
$$

Note that $f$ is continuous in $(\beta, t)$ and is $C^{1}$ in $t$ for each fixed $\beta \in \Gamma$. Moreover, for each $u<T$, there is a compact set $K_{u} \subseteq \Gamma$ such that

$$
F(t):=\min _{\beta \in \Gamma} f(\beta, t) \equiv \ell_{g(t)}(\Gamma)
$$

is attained in $K_{u}$ for $0 \leq t \leq u$. It follows therefore from (3.2) that the lower derivate

$$
\bar{D} F(t):=\liminf _{s \rightarrow t} \frac{F(s)-F(t)}{s-t}
$$

satisfies

$$
\bar{D} F(t) \geq-\frac{(n-1) C_{n}^{2}}{F(t)}, \quad 0 \leq t<T
$$

Hence as in $\S 3$ of [3], we conclude that

$$
(F(t))^{2}+2(n-1) C_{n}^{2} t
$$

is nondecreasing, as required.

The preceding lemma yields a monotonicity result dual to Lemma 1. 
Lemma 4. If $\left(M^{n}, g(t): 0 \leq t<T\right)$ is a solution of the Ricci flow, then $m_{g(t)}(\Gamma)$ is non-decreasing.

Proof. By Lemma 3, we have

$$
\left(m_{g(t)}(\Gamma)\right)^{2}=\liminf _{k \rightarrow \infty} \frac{\left(\ell_{g(t)}(k \Gamma)\right)^{2}}{k^{2}} \geq \liminf _{k \rightarrow \infty} \frac{\left(\ell_{g(s)}(k \Gamma)\right)^{2}-C t}{k^{2}}=\left(m_{g(s)}(\Gamma)\right)^{2}
$$

whenever $0 \leq s \leq t<T$.

To exploit Lemma 4 , we need to know when $m_{g(0)}(\Gamma)$ is nonzero. Let $\eta(\Gamma)$ denote the image of $\Gamma$ in $H_{1}\left(M^{n} ; \mathbb{R}\right)$.

Lemma 5. If $\left(M^{n}, g\right)$ is a Riemannian manifold and $\Gamma \in \operatorname{Free}\left(M^{n}\right)$ is a free homotopy class such that $\eta(\Gamma)$ is nonzero, then $m_{g}(\Gamma)>0$.

Proof. Since $\eta(\Gamma) \neq 0$, there exists $\Phi \in H^{1}\left(M^{n} ; \mathbb{R}\right)$ such that $\langle\Phi, \eta(\Gamma)\rangle>0$. For any $\phi \in \Phi$ and any curve $\gamma \in k \Gamma$, we have

$$
\langle\Phi, \eta(k \Gamma)\rangle=\int_{\gamma} \phi \leq\|\phi\| \cdot \operatorname{length}_{g}(\gamma) .
$$

Taking the infimum over $\phi$ and $\gamma$ yields

$$
\langle\Phi, \eta(k \Gamma)\rangle \leq N_{g}(\Phi) \cdot \ell_{g}(k \Gamma) .
$$

Hence

$$
m_{g}(\Gamma)=\liminf _{k \rightarrow \infty} \frac{\ell_{g}(k \Gamma)}{k} \geq \liminf _{k \rightarrow \infty} \frac{\langle\Phi, \eta(k \Gamma)\rangle}{k N_{g}(\Phi)}=\frac{\langle\Phi, \eta(\Gamma)\rangle}{N_{g}(\Phi)}>0 .
$$

These observations lead to another proof of the main result of this paper.

Second proof of Theorem 1. Let $\left(M^{n}, g(t): 0 \leq t<T\right)$ be a solution of the Ricci flow, and let $\alpha \in H_{1}\left(M^{n} ; \mathbb{Z}\right)$ be an element of infinite order. Then there exists a free homotopy class $\Gamma \in \operatorname{Free}\left(M^{n}\right)$ whose image in $H_{1}\left(M^{n} ; \mathbb{Z}\right)$ is $\alpha$. Clearly, $L_{\alpha}(g(t))=\ell_{g(t)}(\Gamma) \geq m_{g(t)}(\Gamma)$. Since $\alpha$ is of infinite order, $\eta(\Gamma) \in H_{1}\left(M^{n} ; \mathbb{R}\right)$ is nonzero. So we can apply Lemmas 4 and 5 to conclude that

$$
L_{\alpha}(g(t)) \geq m_{g(t)}(\Gamma) \geq m_{g(0)}(\Gamma)>0 .
$$

\section{Concluding remarks}

Although one expects Ricci flow evolutions to encounter finite-time singularities for a large class of initial Riemannian manifolds, the main result of this paper shows that there are topological restrictions on the geometry of such singularities. Motivated by this observation, we pose the following problems. ${ }^{1}$

\footnotetext{
${ }^{1}$ We wish to thank the referee for thoughtful comments regarding these questions.
} 
Problem 1. Let $\left(M^{n}, g_{j}(t)\right)$ be a dilation sequence as in (1.2). Suppose that the rescaling factors $\lambda_{j} \rightarrow \infty$ and that $\left(M^{n}, g_{j}(t)\right)$ converges smoothly in the pointed sense to a limit $\left(N^{n}, h(t)\right)$. Show that the image of $H^{1}\left(N^{n} ; \mathbb{Z}\right)$ in $H^{1}\left(M^{n} ; \mathbb{Z}\right)$ under the natural map is finite. (Following Theorem 1, the idea is that any element of infinite order will be expanded to infinite length.)

Problem 2. If $\pi_{1}\left(M^{n}\right)$ is finite, will any solution $\left(M^{n}, g(t)\right)$ of the Ricci flow become singular in finite time?

Problem 3. Let $\left(M^{n}, g(t)\right)$ be a solution of the Ricci flow. The techniques of Section 3 (in particular Lemma 4) give a lower bound for the length of any curve representing a free homotopy class $\Gamma \in \operatorname{Free}\left(M^{n}\right)$ such that $m_{g(0)}(\Gamma)$ is nonzero. Lemma 5 provides a sufficient condition for this but does not apply in case $M^{n}$ has vanishing first homology. In general, if $\left(M^{n}, g\right)$ is a given Riemannian manifold and $\Gamma \in \operatorname{Free}\left(M^{n}\right)$ is a free homotopy class, when is $m_{g}(\Gamma)$ positive?

\section{References}

[1] H.-D. Cao, B. Chow, Recent developments on the Ricci flow, Bull. Amer. Math. Soc. (N.S.) 36 (1999), 59-74.

[2] S.-C. Chu, Monotonicity formulas and matrix-type inequalities for the harmonic map flow on a manifold evolving by Ricci flow, preprint.

[3] R. Hamilton, Four-manifolds with positive curvature operator, J. Differential Geom. 24 (1986), 153-179.

[4] - The formation of singularities in the Ricci flow, Surveys in differential geometry, Vol. II (Cambridge, MA, 1993), 7-136, International Press, Cambridge, MA, 1995.

[5] _ A compactness property for solutions of the Ricci flow, Amer. J. Math. 117 (1995), 545-572.

[6] , Non-singular solutions of the Ricci flow on three-manifolds, Comm. Anal. Geom. 7 (1999), 695-729.

[7] William P. Thurston, Three-dimensional manifolds, Kleinian groups and hyperbolic geometry, Bull. Amer. Math. Soc. (N.S.) 6 (1982), 357-381.

ETH Zentrum, CH-8092 ZÜRICH, Switzerland.

E-mail address: tom.ilmanen@math.ethz.ch

Department of Mathematics, University of Iowa, 14 Maclean Hall, Iowa City, IA 52242 , U.S.A.

E-mail address: dknopf@math.uiowa.edu 